\title{
Iron-Catalyzed Coupling Reactions of Vinylic Chalcogenides with Grignard Reagents
}

\author{
Claudio C. Silveira,* Samuel R. Mendes and Lucas Wolf \\ Departamento de Química, Universidade Federal de Santa Maria, CP 5001, \\ 97105-970 Santa Maria-RS, Brazil
}

\begin{abstract}
Este trabalho descreve um novo método para a reação de acoplamento entre selenetos e teluretos vinílicos e reagentes de Grignard, catalisada por $\mathrm{Fe}(\mathrm{acac})_{3}$ e à temperatura ambiente. A reação ocorre com retenção da configuração, fornecendo os respectivos alquenos em bons a excelentes rendimentos. Este método também é eficiente para a reação de acoplamento de calcogenetos bisvinílicos com reagentes de Grignard.
\end{abstract}

A general new method for the cross-coupling reaction between vinylic selenides and tellurides and Grignard reagents catalyzed by $\mathrm{Fe}(\mathrm{acac})_{3}$ at room temperature is described. This reaction proceeded with retention of configuration, providing the respective alkenes in good to excellent yields. This method is also efficient for the coupling reaction of divinyl chalcogenides with Grignard reagents.

Keywords: iron-catalyzed, vinylic chalcogenides, cross-coupling, Grignard reagents

\section{Introduction}

Organochalcogenium compounds became the key component of a variety of versatile and useful reagents for organic synthesis. The multiple applications of organochalcogenium chemistry have been well described in a number of review articles ${ }^{1-6}$ and books. ${ }^{7-11}$ Functionalized alkynyl $^{12-18}$ and alkenyl ${ }^{19-23}$ chalcogenides have a great potential in organic synthesis, since they are valuable intermediates for the selective preparation of several organic compounds.

Among the many applications of vinylic selenides, divinylic selenides and vinylic sulfides, the cross-coupling reaction with Grignard reagents catalyzed by $\mathrm{Ni}^{18,24-27}$ and $\mathrm{Pd}^{28,29}$ to give the corresponding cross-coupled products, has been described. On the other hand, $\mathrm{Pd}^{19,30}$ and $\mathrm{Ni}^{24,31-33}$ catalyzed cross coupling reactions and tellurium/metal exchange reactions ${ }^{34-42}$ are demonstrative of the usefulness of vinylic tellurides.

Transition-metal-catalyzed $\mathrm{C}-\mathrm{C}$ bond coupling reactions are very important in many areas of science. ${ }^{43,44}$ Most current methods require expensive transitionmetal catalysts and ligands. However, in the last years

*e-mail: silveira@quimica.ufsm.br
Fe-catalyzed $\mathrm{C}-\mathrm{C}$ bond cross coupling reactions of vinylic substrates and Grignard reagents became a subject of intense interest. ${ }^{45-51}$ The vinylic counterpart is quite broad in scope, since vinylic halides, triflates, sulfonates, tosylates and enol phosphates can be used. ${ }^{45-51}$ In continuation to our interest on the synthesis and synthetic applications of vinylic chalcogenides ${ }^{52-58}$ we decided to study the feasibility of their use in cross coupling reaction with Grignard species catalyzed by iron. To the best of our knowledge, iron catalysts have never been used for the coupling of vinylic selenides and tellurides as electrophiles. The possible use of iron catalysts would represent a great improvement over the high cost of palladium precursors and from the concerns about the toxicity of nickel salts. In light of the above comments it is of interest to design a simple, efficient, and versatile method for the stereoselective coupling of vinylic selenides and tellurides with Grignard reagents catalyzed by iron, aiming to the construction of olefins with defined stereochemistry.

\section{Results and Discussion}

Here we describe our results on the reaction of vinylic selenides and tellurides with Grignard reagents under the catalysis of iron species. The first reactions were performed 
using $\left[\mathrm{Fe}(\mathrm{acac})_{3}\right]$, (acac: acetylacetonate), since it is a very stable, cheap and easy to prepare catalyst. ${ }^{59}$ The initial studies were focused on the development of an optimum set of reaction conditions for the coupling reactions of vinylic chalcogenides with Grignard reagents. We chose (E)-phenyl(styryl)tellane (1d) and a THF solution of $n$-octyl magnesium bromide as starting materials, searching for the best solvent and the amount of $\mathrm{Fe}(\mathrm{acac})_{3}$, performing the reactions at room temperature (Tables 1 and 2).

Table 1. Optimization of reaction conditions: effect of solvent ${ }^{\mathrm{a}}$

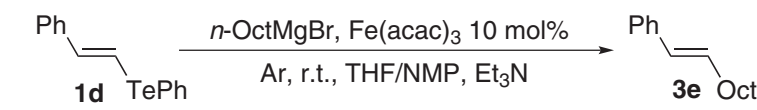

\begin{tabular}{lccc}
\hline entry & Solvent & time $(\mathrm{h})$ & ${\text { Yield }(\%)^{\mathrm{b}}}^{\mathrm{b}}$ \\
\hline 1 & Benzene & 3 & 12 \\
2 & THF & 3 & 41 \\
3 & NMP & 3 & 6 \\
4 & THF/NMP $(3: 1)$ & 2 & 82 \\
\hline
\end{tabular}

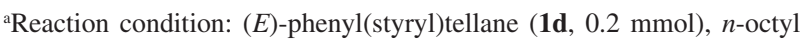
magnesium bromide (1 mmol), $\mathrm{Et}_{3} \mathrm{~N}(0.5 \mathrm{~mL}), \mathrm{Fe}(\mathrm{acac})_{3}(10 \mathrm{~mol} \%)$ and solvent $(4 \mathrm{~mL})$. ${ }^{\mathrm{b}}$ Isolated yield.

At first, we found that by using $10 \mathrm{~mol} \%$ of $\mathrm{Fe}(\mathrm{acac})_{3}$, in benzene, the $(E)$-dec-1-enylbenzene (3e) was obtained in $12 \%$ yield after stirring for $3 \mathrm{~h}$ (Table 1, entry 1 ). We also evaluated other solvents, such as THF and NMP (Table 1, entries 2 and 3). The best yield was obtained when a mixture of THF/NMP (3:1) was used as solvent, and the product 3e was obtained in $82 \%$ yield, after stirring for $2 \mathrm{~h}$ at room temperature (Table 1, entry 4 ). We observed a beneficial effect of the addition of triethylamine as a ligand, in accordance with our own previous observations on the coupling reactions of vinylic chalcogenides catalyzed by nickel. ${ }^{27,33}$ Also, the addition of amine ligands with improved activity on iron cross-coupling have also been observed. ${ }^{60}$

Table 2. Optimization of reaction conditions: effect of amount of catalyst ${ }^{\mathrm{a}}$

\begin{tabular}{|c|c|c|c|}
\hline \multirow{2}{*}{${ }^{\mathrm{Ph}}{ }_{\text {1d }}$} & \multicolumn{2}{|c|}{$n$-OctMgBr, $\mathrm{Fe}(\mathrm{acac})_{3} 10 \mathrm{~mol} \%$} & \multirow{2}{*}{$\stackrel{\mathrm{Ph}}{\overline{3 e} \mathrm{Oct}}$} \\
\hline & TePh & $=/ N M P, \mathrm{Et}_{3} \mathrm{~N}$ & \\
\hline entry & $\mathrm{Fe}(\mathrm{acac})_{3}(\mathrm{~mol} \%)$ & time $(\mathrm{h})$ & Yield $(\%)^{\mathrm{b}}$ \\
\hline 1 & - & 3 & - \\
\hline 2 & 5 & 3 & 48 \\
\hline 3 & 10 & 2 & 82 \\
\hline 4 & 20 & 2 & 82 \\
\hline
\end{tabular}

aReaction condition: (E)-phenyl(styryl)tellane (1d, 0.2 mmol), $n$-octyl magnesium bromide $(1 \mathrm{mmol}), \mathrm{Et}_{3} \mathrm{~N}(0.5 \mathrm{~mL})$ and THF/NMP (3:1, $4 \mathrm{~mL})$. 'isolated yield.
Then, the effect of the amount of the catalyst was evaluated. When the reaction was carried out without catalyst no product was obtained (Table 2 , entry 1 ). The use of $5 \mathrm{~mol} \%$ of $\mathrm{Fe}(\mathrm{acac})_{3}$ produced $\mathbf{3 e}$ in $48 \%$ yield (Table 2, entry 2). The use of larger amounts of catalyst had no effect on the yield of reaction and the required time to completion of reaction was the same (Table 2, entry 4). Thus, the best conditions for the coupling reaction were the use of $\mathrm{Fe}(\mathrm{acac})_{3}(10 \mathrm{~mol} \%)$, THF/NMP (3:1, $\left.4 \mathrm{~mL}\right), \mathrm{Et}_{3} \mathrm{~N}$ $(0.5 \mathrm{~mL})$, vinylic chalcogenide $(0.2 \mathrm{mmol})$ and Grignard reagents $(1 \mathrm{mmol})$, Scheme 1.

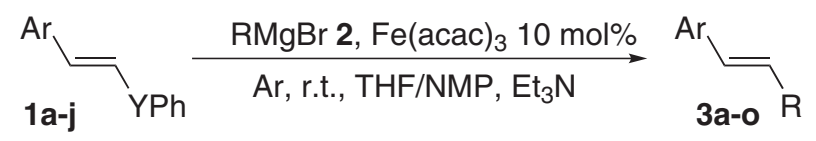
$\mathrm{Y}=\mathrm{Se}, \mathrm{Te}$

Scheme 1.

With these optimized conditions in hand, we next extended to some other examples to find out the scope and limitations of the present method. As can be seen in Tables 3 and 4, these conditions showed to be quite general, and a series of vinylic selenides and tellurides gave the desired products in good yields. Interestingly, the reaction proved to be also efficient for the coupling reaction between divinylic chalcogenides and Grignard reagents. The use of divinylic selenides and tellurides in cross-coupling reactions is particularly interesting since both organyls linked to selenium and tellurium atoms could

Table 3. Fe-Catalyzed cross-coupling of divinyl selenides and tellurides with Grignard reagents

\begin{tabular}{|c|c|c|c|c|c|c|}
\hline entry & Y & Substrate & $\mathrm{R}$ & Product & time (h) & Yield $(\%)^{\mathrm{a}}$ \\
\hline & $\mathrm{Se}$ & $\mathbf{4 a}(Z / Z)$ & $\mathrm{Bu}$ & 3a $(Z)$ & 2.0 & 62 \\
\hline & $\mathrm{Se}$ & $\mathbf{4 a}(Z / Z)$ & Oct & 3b $(Z)$ & 2.0 & 67 \\
\hline & $\mathrm{Se}$ & $\mathbf{4 a}(Z / Z)$ & $\mathrm{Ph}$ & 3c $(Z)$ & 3.0 & 66 \\
\hline & $\mathrm{Se}$ & $4 \mathbf{b}(E / E)$ & $\mathrm{Bu}$ & 3d $(E)$ & 2.0 & 69 \\
\hline & $\mathrm{Se}$ & $4 \mathbf{b}(E / E)$ & Oct & $3 \mathbf{e}(E)$ & 2.0 & 75 \\
\hline & $\mathrm{Se}$ & $4 \mathbf{b}(E / E)$ & $\mathrm{Ph}$ & $\mathbf{3 f}(E)$ & 2.0 & 66 \\
\hline & $\mathrm{Te}$ & $\mathbf{4 c}(Z / Z)$ & $\mathrm{Bu}$ & 3a $(Z)$ & 1.5 & 70 \\
\hline & $\mathrm{Te}$ & $4 \mathbf{c}(Z / Z)$ & Oct & 3b $(Z)$ & 2.0 & 72 \\
\hline & $\mathrm{Te}$ & $\mathbf{4 c}(Z / Z)$ & $\mathrm{Ph}$ & 3c $(Z)$ & 1.5 & 64 \\
\hline 10 & $\mathrm{Te}$ & $\mathbf{4 d}(E / E)$ & $\mathrm{Bu}$ & $\mathbf{3 d}(E)$ & 2.0 & 62 \\
\hline 11 & $\mathrm{Te}$ & $\mathbf{4 d}(E / E)$ & Oct & $\mathbf{3 e}(E)$ & 2.0 & 67 \\
\hline 12 & $\mathrm{Te}$ & $4 \mathbf{d}(E / E)$ & $\mathrm{Ph}$ & $\mathbf{3 f}(E)$ & 3.0 & 66 \\
\hline
\end{tabular}

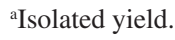


Table 4. Fe-Catalyzed cross-coupling of vinylic selenides and tellurides with Grignard reagents

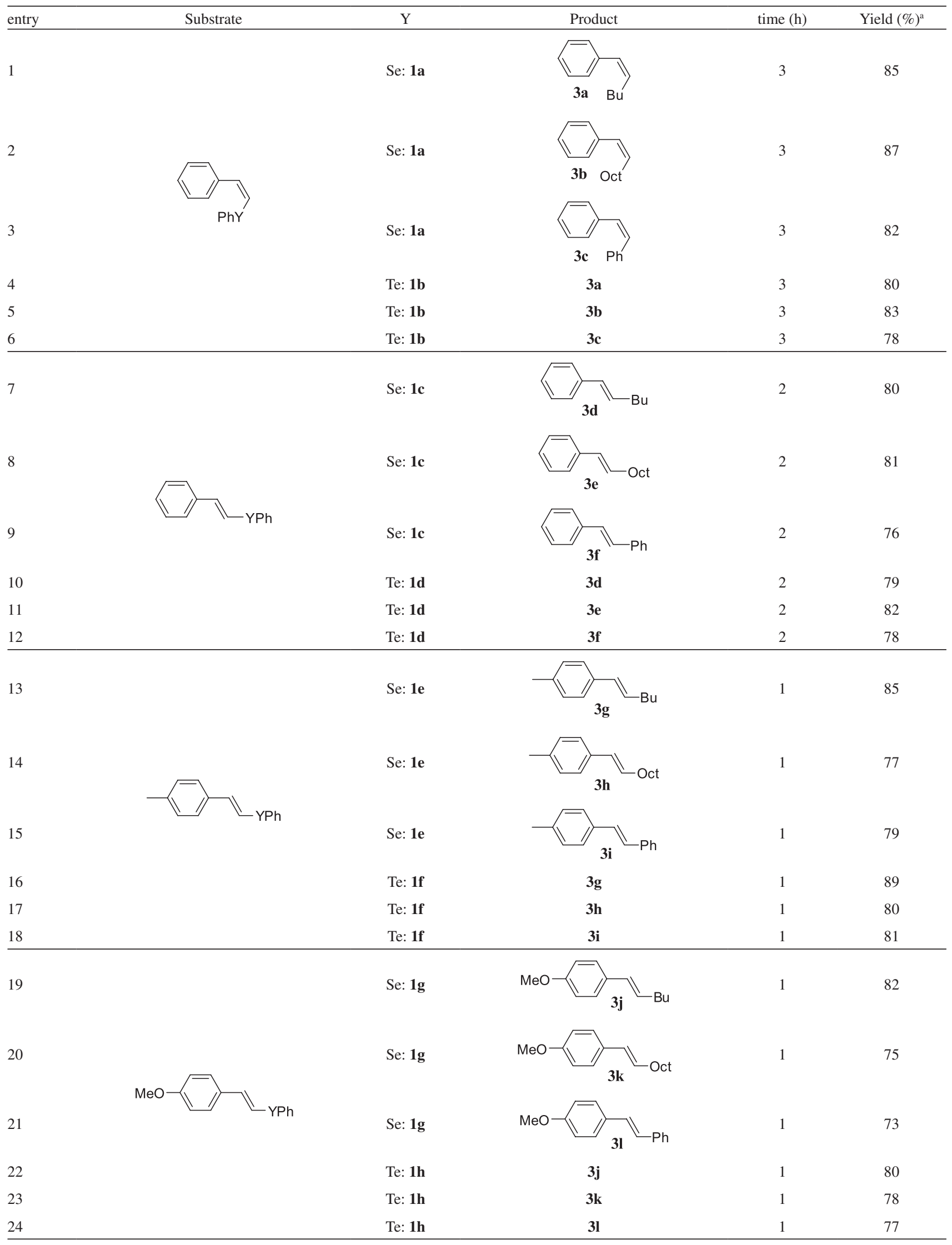


Table 4. continuation

(1):

asolated yield.

eventually be transferred. ${ }^{27,33}$ The iron catalyzed crosscoupling reaction between divinylic chalcogenides and Grignard reagents were carried out at room temperature under standard established conditions and the alkenes $\mathbf{3}$ were obtained in moderate to good yields after stirring for $1.5-3 \mathrm{~h}$ (Table 3). The only difference in this case was the need to increase the amount of the iron catalyst to $20 \mathrm{~mol} \%$ in order to have good yields.

As can be seen in Table 4, the yields from the vinylic chalcogenides were higher than from divinylic chalchogenides. The highest yield was $89 \%$ of the coupling product $\mathbf{3 g}$ using $n$-butyl magnesium bromide and $(E)$-(4methylstyryl)(phenyl)telluride (Table 4, entry 16). For all the studied examples, the alkenes 3 were obtained in good to excellent yields (73-89\%) after stirring at room temperature for 1 to $3 \mathrm{~h}$. The use of aromatic Grignard reagents afforded the corresponding alkenes in lower yields than aliphatic Grignard reagents, and the formation of biphenyl was a competitive reaction. In all cases, no mixture of isomers were observed, the stereochemistry of products was the same as the starting material due to a complete retention of configuration in this type of reaction.

The starting vinylic and divinylic selenides and tellurides have been prepared by procedures previously described or by methods under study in our laboratory. ${ }^{38,61-64}$

Analysis of the ${ }^{1} \mathrm{H}$ NMR and ${ }^{13} \mathrm{C}$ NMR spectra showed that all alkene compounds presented data in full agreement with their assigned structures. The stereochemistry of the obtained alkenes was easily established. As an example, the ${ }^{1} \mathrm{H}$ NMR spectrum of compound $\mathbf{3 d}$ showed a double triplet at $6.21 \mathrm{ppm}$ with coupling constants of 16.0 and $6.6 \mathrm{~Hz}$, typical of trans relationship between hydrogens. The other vinylic hydrogen resonates at $6.37 \mathrm{ppm}$ as a doublet, with a coupling constant of $16.0 \mathrm{~Hz}$. The other signs are in the region between 7.5-7.0 ppm (aromatic hydrogens) and 2.5-0.5 ppm (aliphatic hydrogens).

\section{Conclusions}

In summary, we have been able to demonstrate that $\mathrm{Fe}(\mathrm{acac})_{3}$ is an effective catalyst for the cross-coupling reaction between vinylic selenides and tellurides with Grignard reagents at room temperature. Our method is simple, general and also proved to be efficient for the coupling reaction of divinylic selenides and tellurides with Grignard reagents, with complete retention of configuration. All coupling products were obtained in good to excellent yields.

\section{Experimental}

\section{General}

All ${ }^{1} \mathrm{H}$ and ${ }^{13} \mathrm{C}$ NMR spectra were recorded at $200 \mathrm{MHz}$ and $50 \mathrm{MHz}$, respectively, on a Bruker DPX 200 instrument, using $\mathrm{CDCl}_{3}$ as solvent. Chemical shifts $(\delta)$ are expressed in 
ppm downfield from tetramethylsilane or $\mathrm{CHCl}_{3}$ as internal standard, and $J$ values are given in Hz. Merck's silica gel (230-400 mesh) was used for flash chromatography. All reactions were performed in flame-dried glassware under a positive pressure of argon. Air and moisture sensitive reagents and solvents were transferred via syringe, and were introduced into reaction vessels through a rubber septum. All chemicals were of reagents grade and, if necessary, were purified in the usual manner prior use.

General procedure for the coupling reaction of vinylic selenides and tellurides $\mathbf{1} \boldsymbol{a}-\mathbf{j}$

In a $25 \mathrm{~mL}$ round-bottom flask under argon was added the solvent (THF/NMP 3:1, $4 \mathrm{~mL}$ ), the vinylic chalcogenide (0.2 mmol), $\mathrm{Et}_{3} \mathrm{~N}(0.5 \mathrm{~mL})$ and $\mathrm{Fe}(\mathrm{acac})_{3}(7 \mathrm{mg}, 10 \mathrm{~mol} \%)$. The mixture was stirred and after 5 min the Grignard reagent $(1 \mathrm{mmol})$ was added dropwise. The reaction was followed by TLC and stirred at room temperature for the time indicated on Table 4. After completion of the reaction, aqueous $\mathrm{NH}_{4} \mathrm{Cl}_{\text {(sat) }}(15 \mathrm{~mL})$ was added and the reaction mixture was extracted with ethyl acetate $(3 \times 15 \mathrm{~mL})$, the organic layer was washed with water and dried over $\mathrm{MgSO}_{4}$. After filtration, the solvent was removed under reduced pressure and the residue was purified by column chromatography on silica gel (ethyl acetate-hexanes, 1:99).

General procedure for the coupling reaction of divinyl chalcogenides $\mathbf{4 a - d}$ and compound $\mathbf{1 k}$

In a $25 \mathrm{~mL}$ round-bottom flask under argon was added the solvent (THF/NMP 3:1, $4 \mathrm{~mL}$ ), the divinylic chalcogenide $(0.2 \mathrm{mmol}), \mathrm{Et}_{3} \mathrm{~N}(0.5 \mathrm{~mL})$ and $\mathrm{Fe}(\mathrm{acac})_{3}$ (14 mg, $20 \mathrm{~mol} \%$ ). The mixture was stirred and after 5 min the Grignard reagent ( $2 \mathrm{mmol}$ ) was added dropwise. The reaction was followed by TLC and stirred at room temperature for the time indicated in Table 3 or Table 4. After completion of the reaction, aqueous $\mathrm{NH}_{4} \mathrm{Cl}_{\text {(sat) }}$ $(15 \mathrm{~mL})$ was added and the reaction mixture was extracted with ethyl acetate $(3 \times 15 \mathrm{~mL})$, the organic layer was washed with water and dried over $\mathrm{MgSO}_{4}$. After filtration, the solvent was removed under reduced pressure and the residue was purified by column chromatography on silica gel (ethyl acetate-hexanes, 1:99).

\section{(Z)-Hex-1-enylbenzene $(3 a)^{65}$}

${ }^{1} \mathrm{H}$ NMR $\left(200 \mathrm{MHz}, \mathrm{CDCl}_{3}\right): \delta 7.36-7.16(\mathrm{~m}, 5 \mathrm{H})$, $6.40(\mathrm{~d}, J 11.6 \mathrm{~Hz}, 1 \mathrm{H}), 5.66(\mathrm{dt}, J 11.6$ and $7.20 \mathrm{~Hz}, 1 \mathrm{H})$, 2.38-2.27 (m, 2H), 1.46-1.25 (m, 4H), 0.89 (t, J $6.6 \mathrm{~Hz}$, $3 \mathrm{H}) ;{ }^{13} \mathrm{C} \mathrm{NMR}\left(50 \mathrm{MHz}, \mathrm{CDCl}_{3}\right): \delta 137.86,133.12,128.72$, $128.05,126.35,125.95,32.14,28.31,22.38,13.89$.
(Z)-Dec-1-enylbenzene $(3 \boldsymbol{b})^{66}$

${ }^{1} \mathrm{H} \mathrm{NMR}\left(200 \mathrm{MHz}, \mathrm{CDCl}_{3}\right): \delta$ 7.36-7.19 (m, 5H), 6.40 (d, $J 11.8 \mathrm{~Hz}, 1 \mathrm{H}), 5.66$ (dt, $J 11.8$ and $7.2 \mathrm{~Hz}, 1 \mathrm{H}), 2.32$ (q, J 7.2 Hz, 2H), 1.45-1.26 (m, 12H), $0.87(\mathrm{t}, J 6.6 \mathrm{~Hz}$, $3 \mathrm{H}) ;{ }^{13} \mathrm{C} \mathrm{NMR}\left(50 \mathrm{MHz}, \mathrm{CDCl}_{3}\right): \delta 137.88,133.22,128.74$, 128.06, 126.37, 125.91, 31.87, 29.98, 29.47, 29.35, 29.26, $28.63,22.65,14.05$.

\section{(Z)-1,2-Diphenylethene $(3 c)^{67}$}

${ }^{1} \mathrm{H} \mathrm{NMR}\left(200 \mathrm{MHz} \mathrm{CDCl}_{3}\right): \delta 7.58-7.17(\mathrm{~m}, 10 \mathrm{H})$, 6.57 (s, 2H); ${ }^{13} \mathrm{C} \mathrm{NMR}\left(50 \mathrm{MHz}, \mathrm{CDCl}_{3}\right): \delta 137.32,130.23$, $128.85,128.17,127.11$.

\section{(E)-Hex-1-enylbenzene $(3 \boldsymbol{d})^{68}$}

${ }^{1} \mathrm{H}$ NMR (200 MHz, $\left.\mathrm{CDCl}_{3}\right): \delta 7.35-7.16(\mathrm{~m}, 5 \mathrm{H}), 6.37$ $(\mathrm{d}, J 16.0 \mathrm{~Hz}, 1 \mathrm{H}), 6.21(\mathrm{dt}, J 16.0$ and $6.6 \mathrm{~Hz}, 1 \mathrm{H}), 2.20(\mathrm{q}$, $J 6.6 \mathrm{~Hz}, 2 \mathrm{H}), 1.48-1.30$ (m, 4H), 0.92 (t, J 7.2 Hz, 3H); ${ }^{13} \mathrm{C}$ $\mathrm{NMR}\left(50 \mathrm{MHz}, \mathrm{CDCl}_{3}\right): \delta 137.93,131.17,129.66,128.43$, 126.71, 125.87, 32.71, 31.51, 22.26, 13.95 .

\section{(E)-Dec-1-enylbenzene $(3 \boldsymbol{e})^{27}$}

${ }^{1} \mathrm{H} \mathrm{NMR}\left(200 \mathrm{MHz} \mathrm{CDCl}_{3}\right): \delta 7.30(\mathrm{~d}, J 7.2,2 \mathrm{H}), 7.24$ $(\mathrm{t}, J 7.2,2 \mathrm{H}), 7.14(\mathrm{t}, J 7.2,1 \mathrm{H}), 6.35(\mathrm{~d}, J 15.6 \mathrm{~Hz}, 1 \mathrm{H})$, $6,19$ (dt, $J 15.6$ and $6.8 \mathrm{~Hz}, 1 \mathrm{H}), 2.19$ (q, $J 6.8 \mathrm{~Hz}, 2 \mathrm{H}$ ), 1.46-1.24 (m, 12H), 0.88 (t, $J 6.8 \mathrm{~Hz}, 3 \mathrm{H}) ;{ }^{13} \mathrm{C}$ NMR $(50$ $\left.\mathrm{MHz}, \mathrm{CDCl}_{3}\right): \delta 137.96,131.09,129.76,128.40,126.68$, $125.89,33.07,31.92,29.53,29.43,29.33,29.28,22.70$, 14.08 .

\section{(E)-1,2-Diphenylethene (3f)}

mp 121-123 ${ }^{\circ} \mathrm{C}$ (lit. $\left.{ }^{27} 122-124{ }^{\circ} \mathrm{C}\right) ;{ }^{1} \mathrm{H}$ NMR (200 MHz, $\left.\mathrm{CDCl}_{3}\right): \delta 7.50(\mathrm{~d}, J 7.0 \mathrm{~Hz}, 4 \mathrm{H}), 7.38-7.21(\mathrm{~m}, 6 \mathrm{H}), 7.10$ $(\mathrm{s}, 2 \mathrm{H}) ;{ }^{13} \mathrm{C} \mathrm{NMR}\left(50 \mathrm{MHz}, \mathrm{CDCl}_{3}\right): \delta 137.36,128.73$, $128.65,127.60,126.51$.

\section{(E)-1-(Hex-1-enyl)-4-methylbenzene $(\mathbf{3 g})^{69}$}

${ }^{1} \mathrm{H}$ NMR (200 MHz, $\left.\mathrm{CDCl}_{3}\right): \delta 7.23(\mathrm{~d}, J 8.0 \mathrm{~Hz}, 2 \mathrm{H})$, 7.08 (d, J 8.0 Hz, 2H), 6.34 (d, J 16.0 Hz, 1H), 6.15 (dt, $J$ 16.0 and $6.8 \mathrm{~Hz}, 1 \mathrm{H}), 2.31$ (s, 3H), 2.19 (q, J 6.8 Hz, 2H), 1.49-1.25 (m, 4H), 0.92 (t, J 7.0 Hz, 3H); ${ }^{13} \mathrm{C}$ NMR (50 $\left.\mathrm{MHz}, \mathrm{CDCl}_{3}\right): \delta 136.39,135.17,130.16,129.49,129.14$, $125.77,32.69,31.59,22.25,21.10,13.95$.

\section{(E)-1-(Dec-1-enyl)-4-methylbenzene $(3 \boldsymbol{h})^{27}$}

${ }^{1} \mathrm{H}$ NMR (200 MHz, $\left.\mathrm{CDCl}_{3}\right): \delta 7.23(\mathrm{~d}, J 8.0 \mathrm{~Hz}, 2 \mathrm{H})$, 7.09 (d, J 8.0 Hz, 2H), 6.34 (d, J 16.0 Hz, 1H), 6.08 (dt, J 16.0 and $6.6 \mathrm{~Hz}, 1 \mathrm{H}), 2.33(\mathrm{~s}, 3 \mathrm{H}), 2.18(\mathrm{q}, J 6.6 \mathrm{~Hz}, 2 \mathrm{H})$, $1.50-1.24(\mathrm{~m}, 12 \mathrm{H}), 0.88(\mathrm{t}, J 6.6 \mathrm{~Hz}, 3 \mathrm{H}) ;{ }^{13} \mathrm{C} \mathrm{NMR}(50$ $\left.\mathrm{MHz}, \mathrm{CDCl}_{3}\right): \delta 136.38,135.14,130.22,129.42,129.13$, 125.75, 33.04, 31.89, 29.70, 29.50, 29.44, 29.29, 22.68, $21.11,14.12$. 
(E)-1-Methyl-4-styrylbenzene (3i)

mp 116-118 ${ }^{\circ} \mathrm{C}$ (lit. $\left.{ }^{27} 117-119^{\circ} \mathrm{C}\right){ }^{1} \mathrm{H}$ NMR $(200 \mathrm{MHz}$, $\left.\mathrm{CDCl}_{3}\right): \delta 7.49-7.20(\mathrm{~m}, 7 \mathrm{H}), 7.14(\mathrm{~d}, J 7.8 \mathrm{~Hz}, 2 \mathrm{H}), 7.05(\mathrm{~s}$, $2 \mathrm{H}), 2.33$ (s, 3H); ${ }^{13} \mathrm{C}$ NMR (50 MHz, $\left.\mathrm{CDCl}_{3}\right): \delta 137.48$, $134.50,132.95,129.37,128.62,127.65,127.37,127.13$, $126.39,126.37,21.23$.

\section{(E)-1-(Hex-1-enyl)-4-methoxybenzene $(\mathbf{3 j})^{69}$}

${ }^{1} \mathrm{H} \mathrm{NMR}\left(200 \mathrm{MHz}, \mathrm{CDCl}_{3}\right): \delta 7.27(\mathrm{~d}, J 8.8 \mathrm{~Hz}, 2 \mathrm{H})$, $6.83(\mathrm{~d}, J 8.8 \mathrm{~Hz}, 2 \mathrm{H}), 6.32(\mathrm{~d}, J 15.7 \mathrm{~Hz}, 1 \mathrm{H}), 6.07(\mathrm{dt}, J$ 15.7 and $6.6 \mathrm{~Hz}, 1 \mathrm{H}), 3.79$ (s, 3H), 2.18 (q, J $6.6 \mathrm{~Hz}, 2 \mathrm{H}$ ), 1.46-1.25 (m, 4H), $0.91(\mathrm{t}, J 6.7 \mathrm{~Hz}, 3 \mathrm{H}) ;{ }^{13} \mathrm{C}$ NMR $(50$ $\left.\mathrm{MHz}, \mathrm{CDCl}_{3}\right): \delta 158.59,130.83,129.05,129.01,126.94$, $113.90,55.27,32.68,31.67,22.26,13.95$.

\section{(E)-1-(Dec-1-enyl)-4-methoxybenzene $(3 \boldsymbol{k})^{27}$}

${ }^{1} \mathrm{H} \mathrm{NMR}\left(200 \mathrm{MHz}, \mathrm{CDCl}_{3}\right): \delta 7.25(\mathrm{~d}, J 8.6 \mathrm{~Hz}, 2 \mathrm{H})$, $6.84(\mathrm{~d}, J 8.6 \mathrm{~Hz}, 2 \mathrm{H}), 6.33(\mathrm{~d}, J 15.7 \mathrm{~Hz}, 1 \mathrm{H}), 6.08(\mathrm{dt}, J$ 15.7 and $6.7 \mathrm{~Hz}, 1 \mathrm{H}), 3.79$ (s, 3H), 2.17 (q, $J 6.7 \mathrm{~Hz}, 2 \mathrm{H})$, 1.35-1.22 (m, 12H), $0.88(\mathrm{t}, J 6.7 \mathrm{~Hz}, 3 \mathrm{H}) ;{ }^{13} \mathrm{C}$ NMR $(50$ $\left.\mathrm{MHz}, \mathrm{CDCl}_{3}\right): \delta 158.58,130.83,129.10,128.98,126.94$, $113.88,55.25,33.02,31.89,29.53,29.29,29.27,29.25$, 22.67, 14.09 .

\section{(E)-1-Methoxy-4-styrylbenzene (3l)}

mp 133-135 ${ }^{\circ} \mathrm{C}$ (lit. $\left.{ }^{27} 133-135^{\circ} \mathrm{C}\right) ;{ }^{1} \mathrm{H}$ NMR $(200 \mathrm{MHz}$, $\left.\mathrm{CDCl}_{3}\right): \delta 7.72(\mathrm{~d}, J 6.8 \mathrm{~Hz}, 2 \mathrm{H}), 7.38(\mathrm{~d}, J 16.4 \mathrm{~Hz}, 1 \mathrm{H})$, 7.33-7.18 (m, 5H), 7.12 (d, J 16.4 Hz, 1H), 6.85 (d, J 8.7, $2 \mathrm{H}), 3.80$ (s, $3 \mathrm{H}) ;{ }^{13} \mathrm{C} \mathrm{NMR}\left(50 \mathrm{MHz}, \mathrm{CDCl}_{3}\right): \delta 159.31$, $137.66,130.16,128.63,128.21,127.71,127.20,126.63$, 126.24, 114.14, 55.32.

\section{(E)-1-Chloro-4-(hex-1-enyl)benzene $(3 \boldsymbol{m})^{69}$}

${ }^{1} \mathrm{H}$ NMR $\left(200 \mathrm{MHz}, \mathrm{CDCl}_{3}\right): \delta$ 7.26-7.20 (m, 4H), $6.32(\mathrm{~d}, J 15.7 \mathrm{~Hz}, 1 \mathrm{H}), 6,18(\mathrm{dt}, J 15.7$ and $6.7 \mathrm{~Hz}, 1 \mathrm{H})$, $2.12(\mathrm{q}, J 6.7 \mathrm{~Hz}, 2 \mathrm{H}), 1.40-1.20(\mathrm{~m}, 4 \mathrm{H}), 0.88$ (t, $J 6.8$ $\mathrm{Hz}, 3 \mathrm{H}) ;{ }^{13} \mathrm{C}$ NMR $\left(50 \mathrm{MHz}, \mathrm{CDCl}_{3}\right): \delta 136.42,132.22$, $131.92,129.99,128.54,127.07,32.68,31.40,22.26,13.93$.

\section{(E)-1-Chloro-4-(dec-1-enyl)benzene $(\mathbf{3 n})^{70}$}

${ }^{1} \mathrm{H} \mathrm{NMR}\left(200 \mathrm{MHz}, \mathrm{CDCl}_{3}\right.$ ): $\delta$ 7.28-7.20 (m, 4H), 6.32 (d, $J 15.9 \mathrm{~Hz}, 1 \mathrm{H}), 6.18(\mathrm{dt}, J 15.9,6.4 \mathrm{~Hz}, 1 \mathrm{H}), 2.18(\mathrm{q}$, $J 6.4 \mathrm{~Hz}, 2 \mathrm{H}), 1.36-1.20$ (m, 12H), 0.87 (t, J $6.7 \mathrm{~Hz}, 3 \mathrm{H}$ ); ${ }^{13} \mathrm{C}$ NMR $\left(50 \mathrm{MHz}, \mathrm{CDCl}_{3}\right): \delta 136.45,132.24,131.99$, $128.55,128.51,127.08,33.02,31.88,29.48,29.29,29.28$, $29.24,22.67,14.09$.

\section{(E)-1-Chloro-4-styrylbenzene (3o)}

mp 129-130 ${ }^{\circ} \mathrm{C}$ (lit. $\left.{ }^{71} 129-131{ }^{\circ} \mathrm{C}\right)$; ${ }^{1} \mathrm{H}$ NMR (200 $\mathrm{MHz}, \mathrm{CDCl}_{3}$ ): $\delta 7.79(\mathrm{~d}, J 7.4 \mathrm{~Hz}, 2 \mathrm{H}), 7.63(\mathrm{~d}, J 8.5 \mathrm{~Hz}$, $2 \mathrm{H}), 7.43-7.29(\mathrm{~m}, 5 \mathrm{H}), 7.19$ (s, $2 \mathrm{H}) ;{ }^{13} \mathrm{C}$ NMR $(50 \mathrm{MHz}$,
$\left.\mathrm{CDCl}_{3}\right): \delta 140.01,137.91,135.52,131,31,129.90,129.55$, $128.24,127.55,127.03,125.50$.

\section{4,4'-Di((E)-but-1-enyl)biphenyl (3p)}

mp 114-117 ${ }^{\circ} \mathrm{C}$; ${ }^{1} \mathrm{H}$ NMR (200 MHz, $\left.\mathrm{CDCl}_{3}\right): \delta 7.53$ (d, $J$ 8.4, 4H), 7.39 (d, $J 8.4,4 \mathrm{H}), 6.41(\mathrm{~d}, J 15.8 \mathrm{~Hz}, 2 \mathrm{H})$, $6.30(\mathrm{dt}, J 15.8$ and $6.4 \mathrm{~Hz}, 2 \mathrm{H}), 2.29-2.21(\mathrm{~m}, 4 \mathrm{H}), 1.11$ $(\mathrm{t}, J 7.2 \mathrm{~Hz}, 6 \mathrm{H}) ;{ }^{13} \mathrm{C} \mathrm{NMR}\left(50 \mathrm{MHz}, \mathrm{CDCl}_{3}\right): \delta 139.16$, $136.92,132.75,128.40,126.87,126.30,26.10,13.64$. MS (70 eV), m/z (Rel. Int. \%): M+ 262 (100), 247 (13), 205 (49), 191 (23), 165 (12), 116 (10), 103 (14), 55 (20).

\section{4,4'-Di((E)-hex-1-enyl)biphenyl (3q)}

mp 125-128 ${ }^{\circ} \mathrm{C} ;{ }^{1} \mathrm{H}$ NMR $\left(200 \mathrm{MHz}, \mathrm{CDCl}_{3}\right): \delta 7.52$ $(\mathrm{d}, J 8.2,4 \mathrm{H}), 7.39(\mathrm{~d}, J 8.2,4 \mathrm{H}), 6.41(\mathrm{~d}, J 16.0 \mathrm{~Hz}, 2 \mathrm{H})$, 6.25 (dt, $J 16.0$ and $6.3 \mathrm{~Hz}, 2 \mathrm{H}), 2.22(\mathrm{q}, J 6.3 \mathrm{~Hz}, 4 \mathrm{H})$, $1.50-1.26(\mathrm{~m}, 8 \mathrm{H}), 0.93(\mathrm{t}, J 6.9 \mathrm{~Hz}, 6 \mathrm{H}) ;{ }^{13} \mathrm{C}$ NMR $(50$ $\left.\mathrm{MHz}, \mathrm{CDCl}_{3}\right): \delta 139.10,136.86,131.30,129.24,126.84$, 126.27, 32.78, 31.52, 22.27, 13.96 .

\section{4,4'-Di((E)-dec-1-enyl)biphenyl (3r)}

mp 128-130 ${ }^{\circ} \mathrm{C}$; ${ }^{1} \mathrm{H}$ NMR $\left(200 \mathrm{MHz}, \mathrm{CDCl}_{3}\right): \delta 7.53$ $(\mathrm{d}, J 8.4,4 \mathrm{H}), 7.40(\mathrm{~d}, J 8.4,4 \mathrm{H}), 6.42(\mathrm{~d}, J 15.8 \mathrm{~Hz}, 2 \mathrm{H})$, 6.26 (dt, $J 15.8$ and $6.2 \mathrm{~Hz}, 2 \mathrm{H}), 2.22(\mathrm{q}, J 6.2 \mathrm{~Hz}, 4 \mathrm{H})$, 1.56-1.27 (m, 24H), $0.88(\mathrm{t}, J 6.7 \mathrm{~Hz}, 6 \mathrm{H}) ;{ }^{13} \mathrm{C}$ NMR $(50$ $\left.\mathrm{MHz}, \mathrm{CDCl}_{3}\right): \delta 139.15,136.93,131.41,129.26,126.88$, 126.30, 33.14, 31.90, 29.51, 29.41, 29.30, 29.26, 22.68, 14.11. MS (70 eV), $\mathrm{m} / \mathrm{z}$ (Rel. Int. \%): M+ 428 (4), 425 (100), 327 (9), 301 (15), 202 (36), 188 (12), 43 (13).

\section{Acknowledgments}

This project is funded by MCT/CNPq, CAPES and FAPERGS.

\section{References}

1. Tang,Y.; Ye, S.; Sun, X. L.; Synlett 2005, 2720.

2. Petragnani, N.; Stefani, H. A.; Tetrahedron 2005, 61, 1613.

3. Ranganathan, S.; Muraleedharan, K. M.; Vaish, N. K.; Jayaraman, N.; Tetrahedron 2004, 60, 5273.

4. Aggarwal, V. K.; Winn, C. L.; Acc. Chem. Res. 2004, 37, 611.

5. Comasseto, J. V.; Barrientos-Astigarraga, R. E.; Aldrichimica Acta 2000, 33, 66.

6. Engman, L.; Acc. Chem. Res. 1985, 18, 274.

7. Petragnani, N.; Stefani, H. A.; Tellurium in Organic Synthesis Best Synthetic Methods, $2^{\text {nd }}$ ed., Academic Press: London, 2007.

8. Stang, P. J.; Zhdankin, V. V. In Comprehensive Organic Functional Group Transformations, Ley, S. V., ed., Elsevier: Cambridge, 2003; Vol. II, Chapter 2. 
9. Whitham, G. H. In Organosulfur Chemistry (Oxford Chemistry Primers, 33); Davies, S. G., ed., Oxford University Press: Oxford, 1995.

10. Patai, S.; Rappoport, Z. eds.; The Chemistry of Organic Selenium and Tellurium Compounds, Wiley: Chichester, 1986, Vol. 1.

11. Irgolic, K. Y. In Houben-Weyl Methods of Organic Chemistry; Klamann, D., ed.; $4^{\text {th }}$ ed., vol. E12b; Georg Thieme: Stuttgart, 1990.

12. Perez-Balado, C.; Markó, I. E.; Tetrahedron 2006, 62, 2331.

13. Bieber, L. W.; da Silva, M. F.; Menezes, P. H.; Tetrahedron Lett. 2004, 45, 2735.

14. Braga, A. L.; Martins, T L.; Silveira, C. C.; Rodrigues, O. E. D.; Tetrahedron 2001, 57, 3297.

15. Su, H.; Yu, W.; Jin, Z.; Tetrahedron Lett. 2001, 42, 3771.

16. Braga, A. L.; Silveira, C. C.; Reckziegel, A.; Menezes, P. H.; Tetrahedron Lett. 1993, 34, 8041.

17. Braga, A. L.; Reckziegel, A.; Menezes, P. H.; Stefani, H. A.; Tetrahedron Lett. 1993, 34, 393.

18. Tingoli, M.; Tiecco, M.; Testaferri, L.; Temperini, A.; Tetrahedron 1995, 51, 4691.

19. Lüdtke, D. S.; Panatieri, R. B.; Braga, A. L.; Zeni, G.; Chem. Rev. 2006, 106, 1032.

20. Araujo, M. A.; Raminelli, C.; Comasseto, J. V.; J. Braz. Chem. Soc. 2004, 15, 358.

21. Silveira, C. C.; Perin, G.; Jacob, R. G.; Braga, A. L.; Phosphorus Sulfur, Silicon Relat. Elem. 2001, 172, 309.

22. Comasseto, J. V.; Ling, L. W.; Petragnani, N.; Stefani, H. A.; Synthesis 1997, 373.

23. Alves, D.; Pena, J. M.; Vieira, A. S.; Botteselle, G. V.; Guadagnin, R. C.; Stefani, H. A.; J. Braz. Chem. Soc. 2009, 20,988 .

24. Gerard, J.; Hevesi, L.; Tetrahedron 2001, 57, 9109.

25. Tingoli, M.; Tiecco, M.; Testaferri, L.; Chianelli, D.; Gazz. Chim. Ital. 1991, 121, 59.

26. Okamura, H.; Miura, M.; Kosugi, K.; Takei, H.; Tetrahedron Lett. 1980, 21, 87.

27. Silveira, C. C.; Santos, P. C. S.; Braga A. L.; Tetrahedron Lett. 2002, 43, 7517.

28. Mehta, V. P.; Sharma, A.; Van der Eycken, E.; Org. Lett. 2008, $10,1147$.

29. Hevesi, L.; Hermans, B.; Allard C.; Tetrahedron Lett. 1994, 36, 6729.

30. Zeni, G.; Braga, A. L.; Stefani, H. A.; Acc. Chem. Res. 2003, 36, 731 .

31. Raminelli, C.; Gargalaka Jr, J.; Silveira, C. C.; Comasseto, J. V.; Tetrahedron 2007, 63, 8801.

32. Raminelli, C.; Gargalaka Jr., J.; Silveira, C. C.; Comasseto, J. V.; Tetrahedron Lett. 2004, 45, 4927.

33. Silveira, C. C.; Braga, A. L.; Vieira, A. S.; Zeni, G.; J. Org. Chem. 2003, 68, 662.
34. Bassora, B. K.; Da Costa, C. E.; Gariani, R. A.; Comasseto, J. V.; Dos Santos, A. A.; Tetrahedron Lett. 2007, 48, 1485, and references therein.

35. Seebach, D.; Beck, A. K.; Chem. Ber. 1975, 108, 314.

36. Kauffman, T.; Angew. Chem., Int. Ed.. 1982, 21, 4110.

37. Barros S. M.; Comasseto, J. V.; Berriel, J.; Tetrahedron Lett. 1989, 30, 7353.

38. Tucci, F. C.; Chieffi, A.; Comasseto, J. V.; Marino, J. P.; J. Org. Chem. 1996, 61, 4975.

39. Terao, J.; Kambe, N.; Sonoda, N.; Tetrahedron Lett. 1996, 37, 4741.

40. Huang, Y. Z.; Mo, X. S.; Synlett 1998, 93.

41. Mo, X. S.; Huang, Y. Z.; Tetrahedron Lett. 1995, 36, 3539.

42. Jang, W. B.; Oh, D. Y.; Lee, C. W.; Tetrahedron Lett. 2000, 41, 5103.

43. Negishi, E. I.; Handbook of Organopalladium Chemistry for Organic Synthesis, Wiley and Sons: New York, 2002.

44. Singh, R.; Sharma, M.; Mamgain, R.; Rawat, D. S.; J. Braz. Chem. Soc. 2008, 19, 357.

45. Bolm, C.; Legros, J.; Le Paith, J.; Zani, L.; Chem. Rev. 2004, 104, 6217, and references therein.

46. Cahiez, G.; Gager, O. ; Habiak, V.; Synthesis 2008, 2636.

47. Cahiez, G.; Duplais, C.; Moyeux, A.; Org. Lett. 2007, 9, 3253.

48. Volla, C. M. R.; Vogel, P.; Angew. Chem., Int. Ed. 2008, 47, 1305.

49. Gogsig, T. M.; Lindhardt, A. T.; Skrydstrup, T.; Org. Lett. 2009, 11, 4886.

50. Scheiper, B.; Bonnekessel, M.; krause, H.; Furstner, A.; J. Org. Chem. 2004, 69, 3943.

51. Le Marquand, P.; Tsui, G. C.; Whitney, J. C. C.; Tam, W.; J. Org. Chem. 2008, 73, 7829.

52. Silveira, C. C.; Rinaldi, F.; Guadagnin, R. C.; Braga, A. L.; Synthesis 2009, 469.

53. Silveira, C. C.; Caliari, V.; Vieira, A. S.; Mendes, S. R.; J. Braz. Chem. Soc. 2007, 18, 1481.

54. Silveira, C. C.; Cella, R.; Vieira, A. S.; J. Organomet. Chem. 2006, 691, 5861.

55. Silveira, C. C.; Braga, A. L.; Guadagnin, R. C.; Tetrahedron Lett. 2003, 44, 5703.

56. Silveira, C. C.; Braga, A. L.; Guerra, R. B.; Tetrahedron Lett. 2002, 43, 3395.

57. Silveira, C. C.; Nunes, M. R. S.; Wendling, E.; Braga, A. L.; J. Organomet. Chem. 2001, 623, 131.

58. Silveira, C. C.; Perin, G.; Braga, A. L.; Petragnani, N.; Synlett 1995, 58.

59. Chaudhuri, M. K.; Ghosh, S. K.; J. Chem. Dalton. Trans. 1983, 839.

60. Bedford, R. B.; Bruce, D. W.; Frost, R. M.; Hird, M.; Chem. Commun. 2005, 4161.

61. Barros, S. M.; Dabdoub, M. J.; Dabdoub, V. B.; Comasseto, J. V.; Organometallics 1989, 8, 1661. 
62. Silveira, C. C. ; Rinaldi, F.; Guadagnin, R. C.; Eur. J. Org. Chem. 2007, 4935.

63. Lee, Chi-Wan; Koh, Y. J.; Oh, D. Y.; J. Chem. Soc. Perkin Trans. 1 1994, 717.

64. Silveira, C. C.; Santos, P. C. S.; Mendes, S. R.; Braga, A. L.; J. Organomet. Chem. 2008, 693, 3787.

65. Cai, M.; Xia, J.; Chen, G.; J. Organomet. Chem. 2004, 689, 2531.

66. Periasamy, M.; Prasad, A. S. B.; Suseela, Y.; Tetrahedron 1995, 51, 2743.

67. Mori, A.; Takahisa, E.; Yamamura, Y.; Kato, T.; Mudalige, A. P.; Kajiro, H.; Hirabayashi, K.; Nishihara, Y.; Hiyama, T.; Organometallics 2004, 23, 1755.
68. Herve, A.; Rodriguez, A. L.; Fouquet, E.; J. Org. Chem. 2005, $70,1953$.

69. Negishi, E.; Takahashi, T.; Baba, S.; Horn, D. E. V.; Okukado, N.; J. Am. Chem. Soc. 1987, 109, 2393.

70. Seo, H.; Hirsch-Weil, D.; Abboud, K. A.; Hong, S.; J. Org. Chem. 2008, 73, 1983.

71. Sugihara, T.; Satoh, T.; Miura, M.; Nomura, M.; Adv. Synth. Catal. 2004, 346, 1765.

Submitted: March 15, 2010

Published online: August 12, 2010 